\title{
Evaluation and management of male lower urinary tract symptoms: treatment patterns and guidelines in a correlation study among Polish urology consultants
}

\author{
Tomasz Borkowski ${ }^{1}$, Tomasz Golabek ${ }^{2}$, Piotr L. Chlosta ${ }^{2}$, Andrzej Borkowski ${ }^{1}$
}

\author{
${ }^{1}$ Department of General, Oncological and Functional Urology, Medical University \\ of Warsaw, Warsaw, Poland \\ ${ }^{2}$ Department of Urology, Jagiellonian University Medical College, Krakow, Poland
}

Submitted: 31 October 2013

Accepted: 28 January 2014

Arch Med Sci 2015; 11, 6: 1340-1351

DOI: 10.5114 /aoms.2015.56358

Copyright @ 2015 Termedia \& Banach

\author{
Corresponding author: \\ Tomasz Golabek MD, PhD \\ Department of Urology \\ Jagiellonian University \\ Medical College \\ 18 Grzegorzecka St \\ 31-531 Krakow, Poland \\ Phone: +48 (12) 4247950 \\ Fax: +48 (12) 4247970 \\ E-mail: elementare@op.pl
}

\begin{abstract}
Introduction: In March 2013, the European Association of Urology (EAU) released a new edition of the guidelines on management of male lower urinary tract symptoms (LUTS), including benign prostatic obstruction. The objective of this study was to evaluate how well the EAU guidelines have been implemented in day-to-day practice by Polish urologists.

Material and methods: A structured questionnaire, which explored how urologists diagnose and manage male lower urinary tract symptoms, was emailed to all certified, actively practicing urologists from a list provided by the Polish Urological Association.

Results: The questionnaire return rate was 33.7\% (135/400). Overall, the median (quartile 1; quartile 3) frequency of correct answers was $65.0 \%$ (58.0\%; $69.0 \%$ ). Analysis of the association of availability and acceptance of the EAU guidelines with question answers showed no pattern. A multivariate regression model showed a positive correlation with regards to correct answers given in the survey and doctors' participation in international congresses $(p=0.018, r=0.181)$. Basket analysis showed the strongest association for those who failed to correctly answer the questions regarding diagnosis of LUTS and overactive bladder (OAB) ( support $=27.41 \%$, confidence $=86.05 \%$ ). Conclusions: Although there is a significant degree of adherence to the 2013 EAU guidelines, some discrepancies between urologists' practice and the recommendations regarding diagnosis and treatment of male LUTS do exist. The data obtained provide valuable benchmarks and also identify possible interventions that may improve the standard of care in this population of patients.
\end{abstract}

Key words: management, prostate, prostatic hyperplasia.

\section{Introduction}

The knowledge about the natural history of benign prostatic hyperplasia (BPH) has broadened immensely over the last 20 years. Consequently, the traditional notion that lower urinary tract symptoms are solely related to BPH has changed. Accumulated evidence on multifactorial etiology of male LUTS has led most experts in the field to regard the entire urinary tract as a single functional unit [1, 2]. Evolution of a more complex approach to the pathogenesis and treatment of LUTS resulted in the formulation of new, more contemporary European Association of Urology (EAU) guidelines on "management of non-neurogenic male LUTS" in 2011, and 
their further modification in 2013: “management of male lower urinary tract symptoms, including benign prostatic obstruction" [3, 4]. These guidelines lay down advice on all aspects of assessment and management of LUTS, including conservative, medical and surgical options.

In many European countries, the EAU guidelines have been adopted and implemented by national urological associations or, alternatively, serve as a basis for the formulation of their own national guidelines by others. In Poland, while several chapters of the EAU guidelines regarding oncological diseases of the urinary tract, as well as urinary incontinence, neurogenic lower urinary tract dysfunction and pain management, have been translated into Polish, others have been accepted as national guidelines without translation.

While the majority of the recommendations in the new 2013 edition of the EAU guidelines on the management of male LUTS have already been in place for some time and, therefore, have become common practice, there are still some new important aspects which require implementation in routine urological practice. At present, little is known about practice patterns of Polish urologists in the management of male lower urinary tract symptoms. Thus, to gain an insight into urologist awareness and approach of the management of LUTS, and in order to evaluate how well the EAU guidelines have been implemented in day-to-day practice, we performed a nationwide survey on the current strategies used by Polish urologists in the diagnosis and management of male patients with lower urinary tract symptoms.

\section{Material and methods}

A 42-point questionnaire was developed using a critical assessment by colleagues within the authors' departments, based on the EAU 2013 guidelines entitled: "Management of male lower urinary tract symptoms (LUTS), including benign prostatic obstruction (BPO)" and following a thorough literature review [4].

The study was conducted between April and July of 2013. The questionnaire was emailed to all certified, actively practicing urologists from the list provided by the Polish Urological Association. The respondents' identity was kept anonymous. Participants received a cover letter, which described the evolution of concepts of the etiology of male LUTS and explained the aim of the survey. The questionnaire consisted of three parts. The questions in part one (16 questions) covered doctors' demographics, use of urological knowledge sources, as well as penetration, awareness and acceptance of the EAU 2013 guidelines. Part two was designed to determine urologists' diagnostic pattern in patients with LUTS and overactive blad- der (OAB) (10 questions), as well as the therapy of those same patients (16 questions) (Appendix 1).

\section{Statistical analysis}

Pearson's $\chi^{2}$ test was used to analyze the association between provision of correct and incorrect answers to the survey questions by the doctors and the accessibility and acceptance of the EAU guidelines.

Spearman's correlation coefficient and multivariate logistic regression analysis were applied to determine the correlation of answering a question correctly and incorrectly with time since having completed the residency program in urology, number of urological journals to which the participant subscribed, number of national and international conferences attended, as well as being an EAU member. The Wald statistic was used to test the significance of the regression coefficient.

The number of doctors who answered correctly within the four specific specialty knowledge domains (diagnosis of LUTS and OAB, treatment of LUTS and OAB) was expressed in percentages, where a $60 \%$ cut off was considered satisfactory for diagnosis and treatment of LUTS. Since there was only one question regarding diagnosis of $O A B$ and one about the treatment of OAB in the survey, a respondent was considered to pass those parts of the test if he/she answered them correctly.

To determine whether choosing the wrong answer within one knowledge domain increased the likelihood of answering incorrectly within other domains, the association rules analysis (a detailed version of market basket analysis) was performed. A $p$ value of $<0.05$ was considered statistically significant. The Statistical Package for the Social Sciences (SPSS), version 19, was used for all statistical analyses.

\section{Results}

Of the 400 certified, actively practicing urologists, registered with the Polish Urological Association, 136 returned the questionnaire. One was discarded because of incomplete details, leaving 135 (33.7\%). Forty-three percent of respondents (58/135) had been certified urologists for more than 15 years, $20 \%(27 / 135)$ for less than 5 years, 20\% (27/135) had been practicing urology as specialists for 10 to 15 years, and $17.0 \%(23 / 135)$ for 5 to 10 years. Urologists worked only in the outpatient department (OPD) in $50.4 \%$ of cases (68/135). The remainder (67/135) practiced in both a urology department and an OPD. Fifty-seven percent of responding doctors (77/135) worked in public outpatient clinics, and 97\% (131/135) provided services within a private setting. The majority of urologists $(74.8 \%, 101 / 135)$ regularly saw more than 20 patients in OPD clinics a day, whereas $23.0 \%$ (31/135) and 2.2\% (3/135) saw between 10 and 20, and less than 10 patients 
per day, respectively. Urological journals, national and international urological conferences, information provided by pharmaceutical company representatives, urological books, and discussion with colleagues served as a source of knowledge for $39.3 \%$ (53/135), 28.1\% (38/135), 22.2\% (30/135), 7.4\% $(10 / 135)$, and $2.9 \%(4 / 135)$ of respondents, respectively. Only $40 \%$ (54/135) of doctors who completed the survey were members of the EAU. Although all of the respondents of the questionnaire were aware of the new 2013 version of the EAU guidelines on the management of male lower urinary tract symptoms, availability of the latest edition among EAU members was $72.2 \%$ (39/135), whereas among the nonEAU members, it was 65.4\% (53/135). One hundred and four doctors who completed the questionnaire (77.0\%) wished to have all chapters of the 2013 EAU guidelines translated into Polish, 3.0\% (4/135) would like to have only selected chapters translated, and only $20.0 \%$ (27/135) did not express the need for the translation. Complete and partial acceptance of the 2013 EAU guidelines among respondents was $46.7 \%$ (63/135) and 52.6\% (71/135), respectively. Only one doctor (0.7\%) did not agree with the European Association of Urology guideline recommendations.

\section{Adherence to the European Association of Urology guidelines in diagnosis of male patients with lower urinary tract symptoms and overactive bladder}

The EAU guidelines recommend: “...Systematic diagnostic work-up should be done by history, validated symptom questionnaires (e.g. IPSS), both ideally proactively, physical examination, urinalysis, blood analysis, ultrasound (US) of the prostate, bladder and kidneys, uroflowmetry and US measurement of post-void residual urine, and bladder diary in cases of urinary frequency or nocturia..."

For the initial diagnostic evaluation of men with LUTS, a thorough urological history was found to be crucial among $97 \%$ of the respondents. However, $44.4 \%$ of them did not routinely quantify symptoms with the International Prostate Symptom Score (IPSS). Similarly, the use of a bladder diary was not a common practice in $40.0 \%$ of doctors, and $8.1 \%$ would use it to evaluate the treatment outcome only.

The most frequently used tests during the initial work-up in men with LUTS in whom digital rectal examination revealed no abnormalities other than benign prostatic enlargement included prostate-specific antigen (PSA) evaluation (60.7\%) and ultrasonographic examination of the urinary tract $(28.1 \%)$. Urinalysis, blood biochemistry (serum creatinine) and uroflowmetry were used by only $3.0 \%, 0.7 \%$ and $1.5 \%$ of respondents, respectively (Figure 1).

The EAU guidelines recommend: “...Benign prostatic obstruction and detrusor overactivity are urodynamic diagnoses. Filling cystometry and pressure-flow measurements are optional tests, usually indicated before surgical treatment in men who have a maximum flow rate $(\mathrm{Qmax})>15 \mathrm{ml} / \mathrm{s}$..."

Sixty-three percent of respondents considered a maximum urine flow rate of less than $10 \mathrm{ml} / \mathrm{s}$

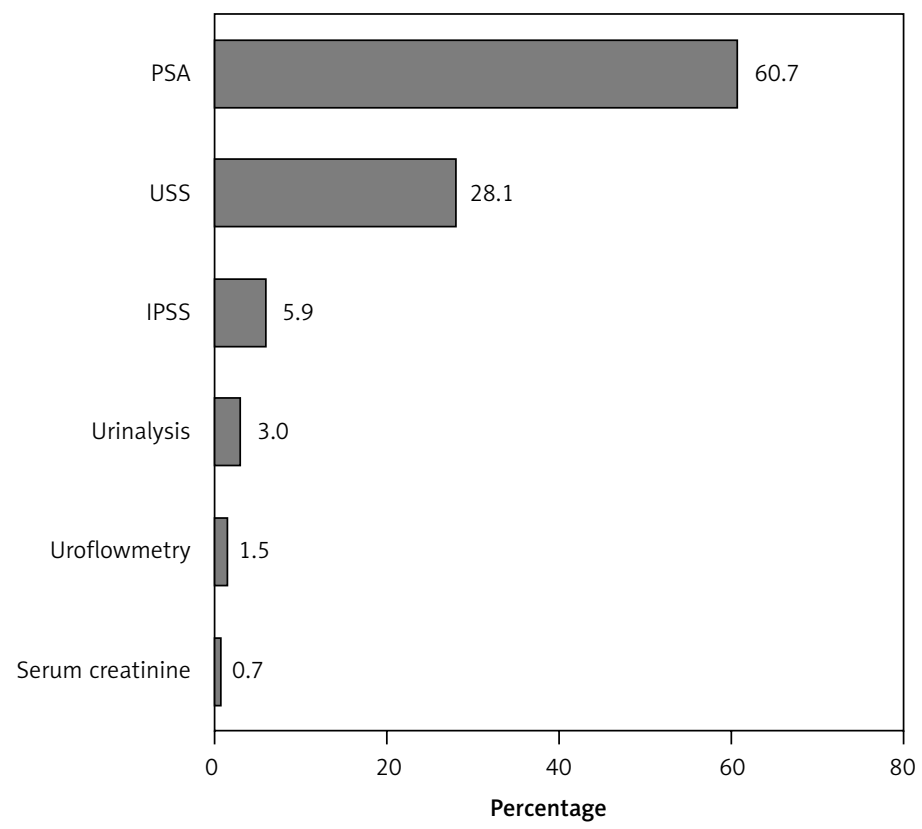

Figure 1. The most frequently used tests during initial work-up in men with LUTS, when digital rectal examination reveals no abnormalities other than benign prostatic enlargement

LUTS - Lower urinary symptoms, PSA - prostate specific antigen, USS - kidney, bladder and prostate ultrasound, IPSS - the International prostate Symptom Score. 
as indicative of bladder outlet obstruction, and $24.4 \%$ of doctors held to a maximum flow of less than $15 \mathrm{ml} / \mathrm{s}$ as the diagnostic threshold. Size of the prostate was considered an important parameter in determining the method of surgical treatment in $43.7 \%$ of respondents. However, $22.2 \%$ of doctors would consider size of the gland as an indication for treatment. To confirm the association between bladder outlet obstruction and benign prostate enlargement, $54.4 \%$ of urologists would perform an ultrasonographic examination of the urinary tract, $50.4 \%$ of doctors would perform uroflowmetry and $21 \%$ would do a filling cystometry and pressure-flow study. Size of the prostate determined on USS would well correspond with the presence of bladder outlet obstruction, as per the opinion of $42.2 \%$ of respondents. In response to the question asking what information the urologist needed in order to diagnose overactive bladder in males, $68.9 \%$ of doctors replied that they required information obtained from the patient history and from objective tests, whereas $14.8 \%$ would base their diagnosis solely on a filling cystometry and pressure-flow study.

\section{Adherence to European Association of Urology guidelines in management of male patients with lower urinary tract symptoms and overactive bladder}

The EAU guidelines recommend: “...Men with mild symptoms are suitable for watchful waiting. Men with LUTS should be offered lifestyle advice prior to or concurrent with treatment... $\alpha_{1}$-Blockers should be offered to men with moderate to mild lower urinary tract symptoms... $\alpha_{1}$-Blockers typically reduce the IPSS, after a run-in period in approximately $35-40 \%$, and increase the maximum urinary flow rate by approximately $20-25 \%$... $\alpha_{1}$-Blockers do not reduce prostate size and do not prevent acute urinary retention..."

Although $10 \%$ of respondents begin pharmacotherapy in patients with minimal or moderate LUTS and $86.7 \%$ of doctors prescribe drugs only in cases of severe symptoms, only $31.9 \%$ of urologists explain to patients that the aim of the treatment is to reduce the frequency of LUTS, whereas $51.9 \%$ of them tell men that it will prevent possible surgical intervention, and a further $14.1 \%$ suggest delaying the surgery as a reason for introducing the medical approach.

Of the $\alpha$-blockers, tamsulosin and doxazosin were used most frequently, by $88.2 \%$ and $11.1 \%$ of urologists, respectively. Although $75.6 \%$ of respondents rightly expected improvement in LUTS when commencing therapy with $\alpha$-blockers, $23.7 \%$ prescribe this class of drugs to reduce the risk of acute urinary retention and surgical treat- ment, and a further $3.0 \%$ of urologists expect prostate size reduction.

The EAU guidelines recommend: “... $5 \alpha$-reductase inhibitors (5ARIs) should be offered to men who have moderate-to severe lower urinary tract symptoms and enlarged prostates $(>40 \mathrm{ml}$ ) or elevated prostate specific antigen concentrations (>1.4-1.6 $\mu \mathrm{g} / \mathrm{l})$. 5ARIs prevent disease progression with regard to acute retention and need for surgery... 5ARIs reduce LUTS (IPSS) by approximately 15-30\%, decrease prostate volume by approximately $18-28 \%$ and increase Qmax by approximately $1.5-2.0 \mathrm{ml} / \mathrm{s}$ in patients with LUTS due to prostatic enlargement... The guidelines committee is unable to make specific recommendations about phytotherapy of male LUTS, because of the heterogeneity of the products and the methodological problems associated with meta-analyses.... Combination treatment with $\alpha_{1}$-blockers and 5ARIs should be offered to men with moderate-to-severe LUTS, enlarged prostates (> $40 \mathrm{ml}$ ), and reduced Qmax..."

When prescribing 5ARI, respondents expected to reduce size of the prostate, decrease the risk of acute urinary retention, and improve the maximal urinary flow rate, as well as LUTS. However, only $45.2 \%$ of urologists correctly anticipated that the size of the gland would be reduced by $15-25 \%$, $53.3 \%$ of doctors knew that the medication would improve symptoms by $15-30 \%$, and $55.6 \%$ of respondents relied on the aforementioned drug to increase the maximal urinary flow rate by $1.5-$ $2.0 \mathrm{ml} / \mathrm{s}$.

Almost all urologists (84.4\%) chose $\alpha$-blockers as the first line treatment option in men with LUTS and a prostate size $<40 \mathrm{cc}$, whereas others prescribed 5ARI monotherapy, $\alpha$-blockers with 5ARIs, phytotherapy, or muscarinic receptor antagonists (Figure 2). $\alpha$-Blockers were the preferred first line treatment option in men with LUTS and prostate size $>40 \mathrm{cc}$, as per the opinion of $48.9 \%$ of respondents, whereas $17.1 \%$ chose 5ARI monotherapy, and $29.6 \%$ prescribed alpha blockers with 5ARIs as the primary management (Figure 3).

The EAU guidelines recommend: "...Muscarinic receptor antagonists (MRA) might be considered in men with moderate to severe LUTS, who have predominantly bladder storage symptoms. Caution is advised in men with bladder outlet obstruction... Increase of PVR urine in men without BOO is minimal and not significantly different compared to placebo..."

Muscarinic receptor antagonists as an acceptable treatment option for storage LUTS was used by $83.7 \%$ of urologists. Additionally, $34.8 \%$ of doctors would consider PVR and prescribe MRA if post-void residual urine volume was less than $150 \mathrm{ml}$, whereas $9.4 \%$ would prescribe that medication regardless of PVR urine volume. 


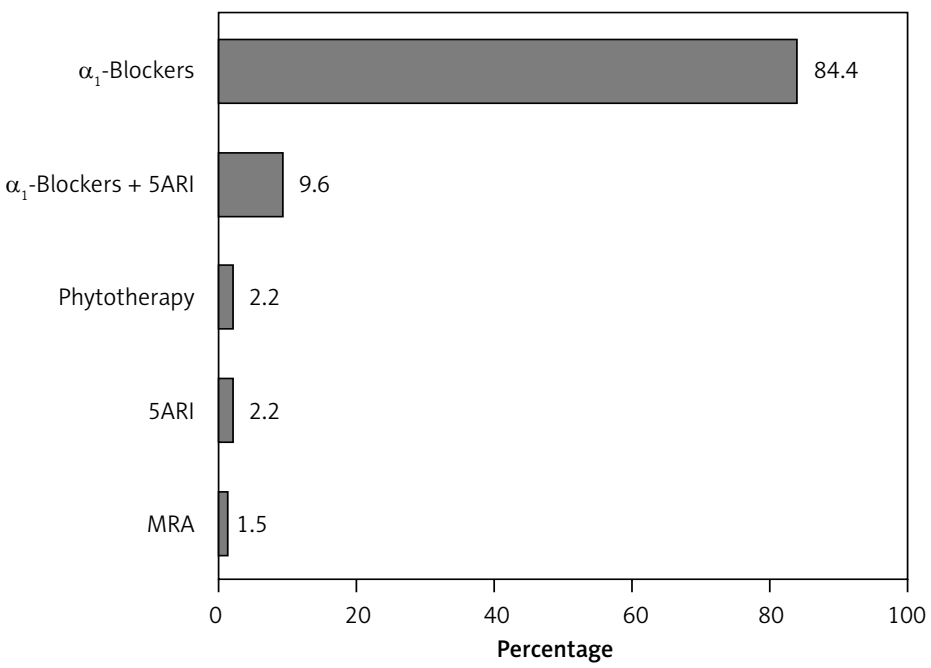

Figure 2. The preferred first line treatment option in men with LUTS and prostate size $<40 \mathrm{cc}$

LUTS - Lower urinary symptoms, cc - cubic centimeters, 5ARI - 5 $\alpha$-reductase inhibitors, MRA - muscarinic receptor antagonists.

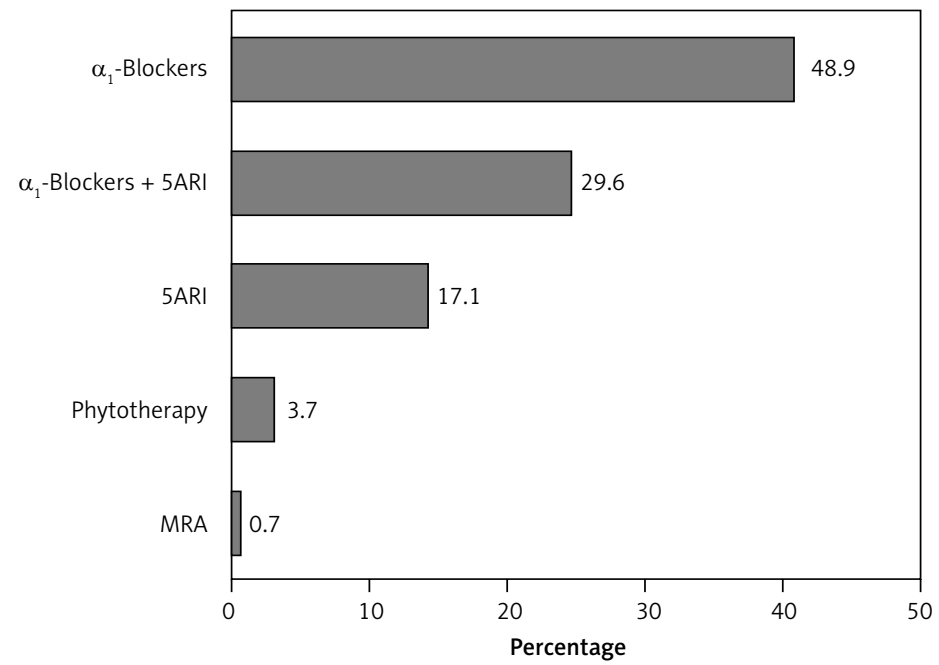

Figure 3. The preferred first line treatment option in men with LUTS and prostate size $>40 \mathrm{cc}$

LUTS - Lower urinary symptoms, cc - cubic centimeters, 5ARI - 5 $\alpha$-reductase inhibitors, MRA - muscarinic receptor antagonists.

The EAU guidelines recommend: “...phosphodiesterase type 5 inhibitors (PDEI-5) reduce moderate to severe male LUTS... To date, only tadalafil $5 \mathrm{mg}$ once daily has been officially licensed for the treatment of male LUTS, with or without erectile dysfunction. Therefore, only tadalafil should be used clinically for the treatment of male LUTS..."

Among 135 urologists participating in the survey, only $10.4 \%$ identified tadalafil as the only phosphodiesterase type 5 inhibitor licensed for the treatment of male LUTS in Europe. In addition, $49.6 \%$ of respondents answered that sildenafil, tadalafil or vardenafil could be used, whereas sildenafil or vardenafil were identified as the only PDEI-5 licensed for treatment by $36.3 \%$ and $3.7 \%$ of doctors, respectively.

The EAU guidelines recommend: “...Refractory urinary retention, recurrent urinary infection, recurrent hematuria refractory to medical treatment with 5ARIs, renal insufficiency due to BPE/BPO, and bladder stones are considered strong indications for surgery... Monopolar TURP is the current surgical standard procedure for men with prostate sizes of 30-80 $\mathrm{ml}$ and moderate-to-severe LUTS secondary to BPO. Monopolar TURP provides subjective and objective improvement rates superior to medical or minimally invasive treatments. However, the morbidity of monopolar TURP is higher than for bipolar TURP, or other minimally-invasive procedures...".

Pharmacotherapy preceded surgical treatment in $80 \%$ of cases. However, only $46.4 \%$ of urologists found renal insufficiency due to benign prostatic obstruction to be an indication for immediate operative procedure without commencing medical therapy. Among urologists who completed the survey, $58.8 \%$ would choose transurethral resection of the prostate (TURP) as a preferred treatment option, while $37.8 \%$ would perform laser vaporization of the prostate, and only $3.7 \%$ would perform open prostatectomy. 


\section{Statistical analysis}

Overall, the median (quartile 1 (Q1); quartile 3 (Q3)) frequency of correct answers was 65.0\% (58.0\%; 69.0\%). The median (Q1; Q3) frequency of correct answers regarding diagnosis and treatment of LUTS was $67.0 \%(56.0 \% ; 73.0 \%)$ and $65.0 \%$ (55.0\%; 73.0\%), respectively. As there was only one question about the diagnosis of $O A B$, and another one about the treatment of $O A B$, no statistical analysis was performed. However, 16.3\% and $80.7 \%$ of respondents, respectively, answered correctly for those questions.

Figure 4 presents the percentage of doctors who passed or failed any of the four domains of the test (diagnosis of LUTS, treatment of LUTS, diagnosis of $O A B$, treatment of $O A B$ ) based on the cut-off value of $60 \%$ of correct answers for passing the test for diagnosis and treatment of LUTS test domains and one correct answer for questions regarding diagnosis of $O A B$ and one about $\mathrm{OAB}$ treatment.

Analysis of the association of availability and acceptance of the 2013 EAU guidelines with answers to the questions within the four tested domains of knowledge showed no pattern. There was only one question about the treatment of mild-to-moderate LUTS, associated with acceptance. There were 60 doctors who accepted the EAU guideline recommendations and answered the above question correctly; only 3 respondents who accepted the EAU guideline recommendations provided an incorrect answer to the question ( $p=0.037$ ). As penetration of the EAU 2013 guidelines was $100 \%$, no analysis was performed for the association with answers.
A multivariate regression model to investigate whether there was a correlation between time since having completed the residency program in urology, number of urological journals to which the respondent subscribed, number of national and international conferences attended, as well as being an EAU member and the correct and incorrect answers was performed. There was a positive correlation with regards to correct answers given in the survey and doctors' participation in international congresses ( $p=0.018, r=0.181)$. However, no other correlation was found.

Basket analysis was performed to determine answer patterns in order to determine whether respondents who failed to answer at least $60 \%$ of questions correctly for one knowledge domain provided incorrect answers to more than 40\% of the questions in the remaining domains. The strongest association was found for those who failed to answer the questions regarding diagnosis of LUTS and a question about diagnosing $\mathrm{OAB}$ correctly (support $=27.41 \%$, confidence $=$ $86.05 \%)$ and for those who incorrectly answered the questions regarding both diagnosis and treatment of LUTS, and a question about treatment of $\mathrm{OAB}$ (support $=11.11 \%$, confidence $=88.24 \%$ ). Table I gives details of the analyzed associations.

\section{Discussion}

From these results, it is clear that the majority of the EAU guidelines on management of male lower urinary tract symptoms, including benign prostatic obstruction, reflect current practice among urologists practicing in Poland. However, there have been several key areas where their

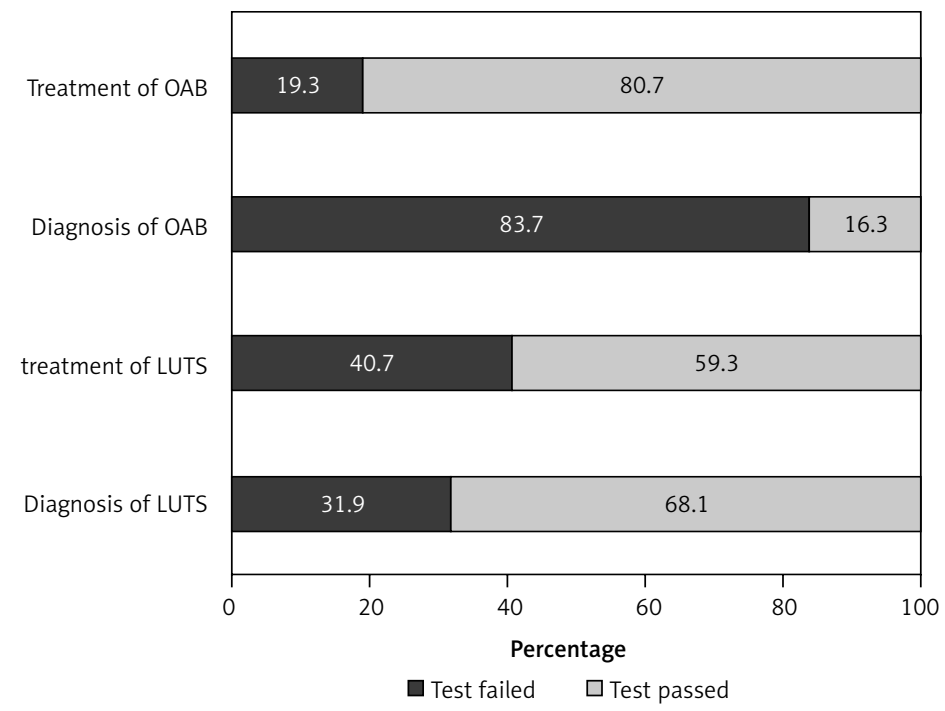

Figure 4. Percentage of doctors who passed or failed any of the four domains of the test (diagnosis of LUTS, treatment of LUTS, diagnosis of OAB, treatment of OAB)

$O A B$ - Overactive bladder, LUTS - lower urinary symptoms; the cut-off value for passing the test is $60 \%$ of correct ansewrs for diagnosis and treatment of LUTS test domain and one correct answer for questions regarding diagnosis of OAB and one about $O A B$ treatment. 
Table I. Basket analysis of incorrect answers

\begin{tabular}{|c|c|c|c|}
\hline Variable 1 & Variable 2 & Support (\%) & Confidence (\%) \\
\hline If D1 fails then & D2 fails & 12.59 & 39.53 \\
\hline If D3 fails then & D4 fails & 17.04 & 20.35 \\
\hline If D1 fails then & D3 fails & 27.41 & 86.05 \\
\hline If D1 + D2 fails then & D3 fails & 11.11 & 88.24 \\
\hline If D1 + D2 fails then & D4 fails & 2.96 & 23.53 \\
\hline If D1 + D2 + D3 fails then & D4 fails & 2.96 & 26.67 \\
\hline
\end{tabular}

bladder, D4 = treatment of overactive bladder.

management differed substantially from guideline recommendations.

The first concerned the initial assessment of a man with LUTS. The EAU guidelines recommend a systematic diagnostic work-up, which should include history, validated symptom questionnaires (e.g. IPSS), physical examination, urinalysis, USS of the prostate, bladder and kidneys, uroflowmetry and USS measurement of post-void residual urine volume, and bladder diary, in the case of urinary frequency, or nocturia [4]. Despite doctors' recognition of the importance of a thorough history, $44.0 \%$ of respondents did not routinely quantify LUTS. In addition, $40.0 \%$ of urologists did not use a bladder diary and a further $8.1 \%$ would use it only to evaluate the treatment outcome. As to the respondents who did not complete the IPSS, the validated questionnaire is a time-consuming tool that offers little advantage in the treatment decision-making process. However, the accumulated evidence in the literature suggests that the IPSS is not a reliable diagnostic tool for LUTS, but serves merely as a measure of LUTS after the diagnosis is established $[5,6]$. Therefore, its significance in the management of men with LUTS cannot be underestimated.

Prevalence of nocturnal polyuria, defined as nocturnal urine volume greater than $33 \%$ of 24 hour volume, can reach up to $89 \%$ of men with LUTS [7]. Diagnosis of this cause of nocturia can be made simply based on the bladder diary [8]. Hence, the initial diagnostic work-up of a patient presenting with nocturia or polyuria should include the completion of a frequency-volume chart.

Similarly to the underuse of the IPSS and bladder diary during the initial assessment of patients with LUTS, respondents would commonly not perform urinalysis and blood analysis (serum creatinine) tests, as well as uroflowmetry. These investigations were only performed by $3.0 \%, 0.7 \%$ and $1.5 \%$ of urologists, respectively. This may have implications for the diagnosis and success of treatment of men with LUTS. It is not clear why these mandatory tests are rarely carried out by the re- spondents. The reported practice was not due to lack of penetration, nor availability or acceptance of the 2013 EAU guideline recommendations. The financial issue should also be disregarded, as those tests are, in fact, inexpensive and readily available.

In this survey, $22.2 \%$ of respondents would consider prostate size as an indication for treatment. Moreover, $42.2 \%$ of urologists believe that the size of the gland corresponds with the bladder outlet obstruction. There is a large body of evidence indicating that prostate size does not correspond with bladder outlet obstruction, and is completely unimportant in determining the need for treatment of male patients with LUTS $[9,10]$. Unfortunately, it is not known why size of the prostate is considered to be an indication for treatment of male LUTS and why many of the doctors believed that it corresponds with bladder outlet obstruction. The reported notions were not due to insufficient penetration, availability, or acceptance of current EAU guidelines, as none of those parameters were as sociated with a failure to provide corrects answers.

In our study, almost all of the urologists commenced pharmacotherapy in patients with moderate or severe lower urinary tract symptoms. However, only $31.9 \%$ of urologists explained to their patients that the aim of the treatment is to reduce LUTS, whereas $51.9 \%$ told the men that the therapy would prevent surgical intervention, and a further $14.1 \%$ gave delay of surgery as a reason for introducing a medical approach. It is unclear why respondents offered limited information to their patients. However, it seems unlikely to be related to lack of awareness about the mechanisms of action of the most commonly prescribed medications, as the majority of respondents correctly identified the expected effects of the most frequently prescribed medications.

The statements concerning the treatment of LUTS with phosphodiesterase type 5 inhibitors were quite interesting. Among 135 urologists participating in the survey, only $10.4 \%$ identified tadalafil as the only PDEI-5 licensed for treatment 
of male LUTS in Europe. The introduction of PDEI-5 for the treatment of moderate-to-severe storage and voiding lower urinary tract symptoms has been a relatively novel approach [11, 12]. This most likely explains the lack of awareness regarding phosphodiesterase type 5 inhibitor use in medical management of male LUTS.

In this study, no association of penetration, availability or acceptance of the 2013 EAU guidelines with answers to the questions was found, except for one question regarding treatment of mild-to-moderate LUTS. Interestingly, in a multivariate regression model, only one weak correlation between potential factors improving access to knowledge and the doctors' answers was identified. Attendance at international, but not national, congresses correlated with correct answers $(p=0.018, r=0.181$ and $p=0.406, r=0.021$, respectively). This finding was enhanced by the fact that $45.9 \%$ of respondents participated in an international meeting only once every several years, $45.9 \%$ of doctors attended a congress annually, and only $8.2 \%$ of urologists took part in 1 to 3 international meetings every year. However, $74.9 \%$ of respondents declared having been present at 1 to 3 national congresses per year, $20.7 \%$ of doctors participated in 4 to 6 domestic meetings annually, $3.7 \%$ of specialists participated once every several years, and only one urologist $(0.7 \%)$ participated less than once every 10 years. However, this may reflect the fact that the 2013 EAU guidelines were introduced in March 2013 and the study was carried out between April and June 2013. Within that period of time, several international meetings took place, including the EAU annual congress, whereas only 3 national meetings that were dedicated exclusively to uro-oncology were organized. On the other hand, as the 2013 EAU guidelines are an update of the previous versions and therefore cover the majority of the recommendations that have been in place for a few years, an insufficient amount of time dedicated for discussion of current EAU recommendations at domestic meetings as well as their quality need to be considered.

In an attempt to identify answer patterns, a basket analysis was performed. This showed that there were not many respondents who provided less than $60 \%$ of correct answers to more than one tested knowledge domain. The strongest relation was seen among those who incorrectly answered questions regarding diagnosis of LUTS and OAB. $27.41 \%$ of doctors incorrectly answered at least $60 \%$ of questions about diagnosis of both LUTS and OAB, and $86.05 \%$ of urologists who provided incorrect answers to more than $40 \%$ of the questions regarding diagnosis of LUTS provided incorrect answers to the question about $O A B$ diagnosis, concurrently.
This survey provides useful insight into current strategies practiced by Polish urologists in the diagnosis and management of male patients with lower urinary tract symptoms. The discrepancies observed between the 2013 EAU guideline recommendations and strategies in diagnosis and management of male LUTS provide a benchmark of practice patterns among Polish urologists. However, some urologists' practice patterns should be altered to improve the standard of care. Further education based on the recent EAU recommendations needs to be continued. Current guidelines on management of male LUTS need to be discussed more often at national meetings and the results of this practice should then be audited. Although this study did not show a correlation between translation of the 2013 EAU guidelines into Polish and answers provided by the doctors who participated in the survey, it seems that translation of the guidelines could be of benefit to at least $80 \%$ of doctors who are not sufficiently familiar with the English language.

This survey is not an epidemiologic study and, thus, it is subject to bias and limitations associated with these study designs. Physicians who completed the survey elected to participate, so they may not be a representative sample of Polish urologists. Moreover, the data used in this survey are based solely on the awareness of respondents, and not on real world scenarios. Therefore, this survey could bear a certain degree of subjectivity. Further, all data were collected through self-administered questionnaires. This could contribute to sampling error, as different survey modes have different sampling errors, response rates, data completeness, and measurement errors [13].

In conclusion, this study provides insight into current strategies used by Polish urologists in the diagnosis and management of male patients with lower urinary tract symptoms. Although there is a significant degree of adherence to the 2013 EAU guidelines, some discrepancies between urologist practice and the recommendations regarding diagnosis and treatment of male LUTS do exist. Obtained data provide valuable benchmarks and identify possible interventions that may be addressed in order to improve the standard of care in this population of patients.

\section{Conflict of interest}

The authors declare no conflict of interest.

\section{References}

1. Chapple CR, Roehrborn CG. A shifted paradigm for the further understanding, evaluation, and treatment of lower urinary tract symptoms in men: focus on the bladder. Eur Urol 2006; 49: 651-8. 
2. Michielsen DP, Coomans D, Van Lersberghe C, Braeckman JG. Comparison of the haemostatic properties of conventional monopolar and bipolar transurethral resection of the prostate in patients on oral anticoagulants. Arch Med Sci 2011; 7: 858-63.

3. Oelke M, Bachmann A, Descazeaud A, et al. Guidelines on the treatment of non-neurogenic male LUTS. Uroweb 2011. Available at: http://www.uroweb.org/gls/pdf/12 Male_LUTS.pdf, Accessed October 17, 2013.

4. Oelke M, Bachmann A, Descazeaud A, et al. (2013) Guidelines on the Management of Male Lower Urinary Tract Symptoms (LUTS), incl. Benign Prostatic obstruction (BPO). European Association of Urology. Available at: http://www.fau.org.ar/pdf/Male_LUTS_fullingles.pdf, Accessed October 31, 2015.

5. Woo HH, Gillman MP, Gardiner R, Marshall V, Lynch WJ. A practical approach to the management of lower urinary tract symptoms among men. Med J Aust 2011; 195: 34-9.

6. Dybowski B, Bres-Niewada E, Radziszewski P. Pressureflow nomogram for women with lower urinary tract symptoms. Arch Med Sci 2014; 10: 752-6.

7. Weiss JP, van Kerrebroeck PE, Klein BM, Nørgaard JP. Excessive nocturnal urine production is a major contributing factor to the etiology of nocturia. J Urol 2011; 186: 1358-63.

8. Dehoorne JL, Walle CV, Vansintjan P, et al. Characteristics of a tertiary center enuresis population, with special emphasis on the relation among nocturnal diuresis, functional bladder capacity and desmopressin response. J Urol 2007; 177: 1130-7.

9. Roehrborn CG. Prostate size: does it matter? Rev Urol 2000; 2: 95-8.

10. Witjes WP, Aarnink RG, Ezz-el-Din K, Wijkstra H, Debruyne EM, de la Rosette JJ. The correlation between prostate volume, transition zone volume, transition zone index and clinical and urodynamic investigations in patients with lower urinary tract symptoms. Br J Uro 1997; 80: 84-90.

11. Porst H, McVary KT, Montorsi F, et al. Effects of once-daily tadalafil on erectile function in men with erectile dysfunction and signs and symptoms of benign prostatic hyperplasia. Eur Urol 2009; 56: 727-35.

12. Roehrborn CG, Kaminetsky JC, Auerbach SM, Montelongo RM, Elion-Mboussa A, Viktrup L. Changes in peak urinary flow and voiding efficiency in men with signs and symptoms of benign prostatic hyperplasia during once daily tadalafil treatment. BJU Int 2010; 105: 502-7.

13. Bowling A. Mode of questionnaire administration can have serious effects on data quality. J Public Health (Oxf) 2005; 27: 281-91. 


\section{Appendix 1}

Part one:

1. How long have you been practicing urology since completing your residency?

$-<5$ years,

$-5-10$ years,

- 10-15 years,

$->15$ years.

2. The type of healthcare sector you work for is:

- private,

- public.

3. Do you see your patients in an outpatient clin-

ic as well as on the wards?

- Yes,

- No.

4. Describe your workplace setting:

- rural area or small town (population less than 50,000),

- town (population between 50,000 and 100,000),

- city (population greater than 100,000).

5. Can you describe the number of daily specialty consultations you usually deliver?

$-<10$,

$-10-20$

$->20$.

6. To how many specialty journals are you currently subscribing?

-0 ,

-1 ,

-2 ,

-3 ,

$$
->3 \text {. }
$$

7. How often do you attend any national conferences or symposia?

- Occasionally, every few years,

-1-3 times per year,

-4-6 times per year,

-7-10 times per year

$->10$ times per year.

8. How often do you attend any international conferences or symposia?

- Occasionally, every few years,

- At least once a year,

- 1-3 times per year,

$->3$ times per year.
9. How do you keep your specialty knowledge up-to-date?

- Books and manuscripts,

- Urology journals,

- Conference proceedings,

- Discussions with colleagues,

- Other.

10. Are you an EAU member?

- Yes,

- No.

11. Are you aware that a new 2013 edition of the EAU guidelines on the management of male lower urinary tract symptoms (LUTS), including benign prostatic obstruction (BPO), has recently been published?

- Yes,

- No.

12. Have you received the EAU 2013 guidelines, because you are an EAU member?

- Yes,

- No.

13. If you are NOT an EAU member, have you still received the EAU 2013 guidelines?

- Yes, the latest version from EAU members,

- Yes, electronic version,

- No.

14. Do you think that the Polish Urological Association ought to translate all available EAU guidelines?

- Yes, all,

- Yes, but not all (if so please describe which ones exactly..........),

- No, I do not mind reading the guidelines in English.

15. How useful do you find the EAU guidelines in your practice?

- I use them regularly,

- I only use them every so often,

- I do not find them useful or transferrable to Polish standards of practice.

16. Do you follow the EAU 2013 guidelines in your daily practice?

- Always,

- Never,

- Sometimes.
Part II: Diagnosis of lower urinary tract symptoms (LUTS) and overactive bladder (OAB)

1. In your patients presenting with LUTS, to which of the following phases are the symptoms they predominantly complain of related?

- Storage,

- Voiding,

- Post-micturition.

2. As per the literature, which two LUTS are considered by the patients as the most bothersome?
- Nocturia,

- Hesitancy,

- Urgency,

- Difficulty passing urine,

- Interrupted urine flow,

- Post-micturition dribble - wetting underwear,

- Incomplete bladder emptying,

- Weak stream of urine.

3. In patients with no suspicious findings on digital rectal examination, and no additional in- 
vestigations available, your initial assessment tool includes which of the following?

- USS,

- Uroflowmetry,

- PSA,

- Serum creatinine or eGFR,

- Mid stream urinalysis,

- Urine culture and sensitivity,

- Micturition diary,

- IPSS questionnaire.

4. With regards to the International Prostate Symptom Score questionnaire (IPSS) please choose one of the options below:

- It is always completed by a patient at the first clinical appointment,

- It is handed over to a patient to complete later at home and to bring back at the next visit,

- I only use it occasionally.

5. What maximum urine flow is the most suggestive of bladder outlet obstruction (BOO)?

$-<5 \mathrm{ml} / \mathrm{s}$,

$-<10 \mathrm{ml} / \mathrm{s}$,

$-<15 \mathrm{ml} / \mathrm{s}$.

6. Does the size of the prostate influence your clinical judgment?

- Yes, it is an indication for treatment,

- Yes, but only if associated with BOO,

- Yes, but only if associated with LUTS,

- Yes, as this can affect the choice of surgical technique,

- It should not affect the clinical judgment with regards to treatment.

7. Do you recommend using a bladder diary to your patients?

- Almost never,

- To each patient with BPH,

\section{Part III: Treatment of LUTS and OAB}

1. What is your next step following the history and investigations of confirmed diagnosis of mild-to-moderate LUTS?

- Watchful waiting,

- Lifestyle modification and diet: cutting the amount of fluids, coffee, alcohol, altering the time of the day when fluids and medications should preferably be taken, bladder training, treating constipation,

- Implementation of phytotherapy (herbal medicines),

- Commencing pharmacotherapy,

- Further management depends on patient's individual preferences,

- Other (describe).

2. What would be your next management step in patients with severe LUTS with the majority of symptoms during the voiding phase?

- Pharmacotherapy,
- Only to patients with LUTS during storage phase,

- Only to assess the therapeutic response.

8. Which of the investigations do you think is required in order to confirm that $\mathrm{BOO}$ is caused by $\mathrm{BPH}$, and that LUTS are associated with the voiding phase?

- USS,

- Uroflowmetry,

- Urethrography,

- Complete urodynamic assessment,

- Other (describe).

9. Which of the following USS findings may suggest $\mathrm{BOO}$ ?

- Upper urinary tract dilatation,

- Post-micturition residual urine volume $>50 \mathrm{ml}$,

- Post-micturition residual urine volume $>100 \mathrm{ml}$,

- Post-micturition residual urine volume $>150 \mathrm{ml}$,

- Prostate size,

- Large middle lobe of the prostate,

- Bladder wall thickness,

- Bladder diverticula,

- Bladder stone.

10. The constellation of symptoms described as overactive bladder is diagnosed based on:

- Predominance of LUTS with urgency during storage phase,

- Exclusion of other disorders presenting with LUTS (i.e. UTI, CIS, radiation cystitis),

- Urodynamic investigation,

- All of the above.
- Surgery,
- Other (describe).

3. Which of the following indications for surgery do you find the most relevant?

- Prostate size,

- Storage phase LUTS,

- Voiding phase LUTS,

- Post-micturition phase LUTS,

- Post-voiding residual volume $>150 \mathrm{ml}$,

- Maximum urinary flow $<10 \mathrm{ml} / \mathrm{s}$,

- Treatment of resistant urinary tract infections (UTIS).

4. With regards to $\mathrm{BPH}$ treatment, when do you obviate medical treatment in favor of surgery as the first line treatment?

- Very large prostate,

- Treatment of refractory UTIs,

- Acute urinary retention,

- Renal failure,

- All of the above. 
5. When prescribing medical treatment for LUTS to a patient, how do you justify your choice over another treatment option(s)?

- To postpone surgical treatment,

- To prevent surgical treatment,

- You concentrate on the treatment of LUTS rather than $\mathrm{BPH}$ progression,

- Other (describe).

6. Which $\alpha_{1}$-blocker do you tend to use the most?

- Doxazosin

- Tamsulosin,

- Alfuzosin,

- Terazosin,

- Other (which one)

7. When using $\alpha_{1}$-blockers, which of the following are you expecting?

- Amelioration of LUTS,

- Improvement in uroflow,

- Reduction in prostate size,

- Preventing progression, thereby reducing the risk of acute urinary retention and possible operative treatment,

- All of the above.

8. When would you decide for $\alpha_{1}$-blocker monotherapy?

- Prostate volume $<30 \mathrm{ml}$, severe LUTS associated with storage phase,

- Prostate volume $<30 \mathrm{ml}$, moderate LUTS associated with storage and voiding phases,

- Prostate volume > $100 \mathrm{ml}$, moderate LUTS associated with storage and voiding phases,

- In every patient with LUTS as the first line treatment.

9. With regards to the $5 \alpha$-reductase inhibitors (5ARIs), which of the following can you expect?

- Amelioration of LUTS,

- Improvement in urinary flow,

- Reduction in prostate size,

- Preventing progression, thereby reducing the risk of acute urinary retention and possible operative treatment,

- All of the above.

10. By how much can the 5ARIs reduce prostate volume?

$-5-10 \%$,

$-10-15 \%$,

$-15-25 \%$

$-25-35 \%$.
11. By how much can the 5ARIs reduce LUTS as per the IPSS?

$-10-15 \%$,

$-15-30 \%$,

$-30-40 \%$.

12. Using the 5ARIs, by how much can the maximum urinary flow be improved?

$-1.0-1.5 \mathrm{ml} / \mathrm{s}$,

$-1.5-2.0 \mathrm{ml} / \mathrm{s}$,

$-2.0-3.0 \mathrm{ml} / \mathrm{s}$,

$-3.0-3.5 \mathrm{ml} / \mathrm{s}$.

13. Which class of medications do you choose for patients with prostate volume of $<40 \mathrm{cc}$ and mild to severe LUTS?

$-\alpha_{1}$-Blockers,

- 5ARIs,

- Combination of $\alpha_{1}$-blocker and 5ARI,

- Antimuscarinics,

- Herbal remedies.

14. Which of the following classes of drugs do you use in men with prostate volume $>40 \mathrm{cc}$ and mild LUTS?

$-\alpha_{1}$-Blockers,

- 5ARIs,

- Combination of $\alpha_{1}$-blocker and 5ARI,

- Antimuscarinics,

- Herbal remedies.

15. It has been shown that in men with concurrent erectile dysfunction and LUTS, who are treated with a combination of $\alpha_{1}$-blocker and 5ARI, which type of 5 phosphodiesterase inhibitor can replace 5ARI?

- Sildenafil,

- Tadalafil,

- Vardenafil,

- All of the above.

16. When do you use antimuscarinics?

- Patients with voiding phase LUTS,

- Patients with storage phase LUTS,

- When post-voiding residual urine volume exceeds $150 \mathrm{ml}$,

- Regardless of the residual volume. 